\title{
Editorial
}

\section{A new p63-deficient mouse model or a fresh look at an old one?}

\author{
D Aberdam ${ }^{1}$ and $\mathrm{R}$ Mantovani ${ }^{\star 2}$ \\ Cell Death and Differentiation (2009) 16, 1073-1074; doi:10.1038/cdd.2009.66
}

The p53-related transcription factor $p 63$ gene encodes six isoforms by the use of two promoters - TA and $\Delta \mathrm{N}-$ and alternative splicing at the $3^{\prime}$-terminus, resulting in $\alpha, \beta$, and $\gamma$ variants. Its critical importance in development has been illustrated by the discovery that dominantly inherited mutations in the p63 gene are found in a number of human ectodermal dysplasias with defects in limb and skin development, ${ }^{1}$ and by the generation of mouse models lacking p 63 by two independent laboratories. Although the strategies for generating these models were different, the phenotypes were very similar: the mice die soon after birth with severe defects in limbs, craniofacial development, and absence of skin. ${ }^{2,3}$ The phenotypes of the different p63-deficient models - three generated by the Bradley laboratory (p63 Brdm1, p63 Brdm2, and p63 Brdm3) as well as the one generated by the McKeon laboratory - were essentially identical in their more evident manifestations. However, differences in the interpretation of the phenotypes of these models have led to different views regarding the role of p63 in epidermal morphogenesis and tumor susceptibility. This is far from unprecedented, especially as different mouse strains (129Sv/B57BL/6 in Bradley's and B57BL/6/BALB/C in McKeon's) and KO strategies were used. McKeon's model was generated in a 'traditional' way, by deleting exons 6-8, coding for the DNA-binding domain (DBD). On the other hand, Mills et al. ${ }^{2}$ generated two mouse strains - Brdm1 and Brdm2 - with targeting vectors that used a gap-repair-dependent mechanism to generate ES cells harboring disrupted p63 alleles; the Brdm1 allele is disrupted within exon 6 of the DBD; the Brdm2 allele is disrupted within intron 10, involving the $3^{\prime}$-region of the gene: the transcript would be devoid of exons 11-14, which are incorporated in the $\alpha$-and $\beta$-isoforms. The same laboratory has reported another mouse strain - Brdm3 - in which a conventional floxed LoxP insertion hits the DBD coding exons 5-7, encoding the majority of the DBD in the case of the p63 Brdm3 allele. The three Brdm mice are phenotypically identical.

In this issue of Cell Death Differentiation, Wolff et al. ${ }^{4}$ performed a set of experiments leading to the conclusion that the Brdm2 mouse model is a partial $\mathrm{KO}:{ }^{4}$ they detected a truncated p63 transcript, reminiscent, but not identical of the endogenous p63 transcript that encodes the p63 $\gamma$-isoform.
The truncated transcript is entirely consistent with the genomic organization of Brdm2 originally reported by Mills et al. $^{2}$ Wolff et al. expressed this transcript in transfections and found that the resulting protein was similar to p63 $\gamma$ in its ability to transactivate an Mdm2 luciferase reporter. Immunohistochemical analysis using the 4A4 and H137 antibodies showed that $p 63$ is clearly present, although the amount of p63 $\gamma$ produced in utero, as determined by western blotting, was barely detectable. Analyses of embryonic sections of McKeon's mice were negative. The $\gamma$-isoforms lack the SAM domain, a protein-protein interaction module present in many developmentally regulated proteins and mutated in Ankyloblepharon Ectodermal Dysplasia and Cleft Lip/ Palate (AEC) patients suffering from skin fragility.

As described in the original paper of Mills et al., ${ }^{2}$ Wolff et al. ${ }^{4}$ report that E18.5 Brdm2 embryos are devoid of limbs and have no epidermis; surprisingly, however, embryos at E15.5 have patchy regions consisting of complete, pluristratified epidermis, and are positive for $\mathrm{p} 63$ and $\mathrm{K} 14$ expression. This is particularly evident in the oral epithelia. This unexpected observation raises several issues. First, the presence of embryonic epidermis at E15.5 leads to the conclusion that the p63 $\gamma$ isoforms are sufficient to allow embryonic epidermal fate, whereas the $\alpha / \beta$-isoforms are necessary for skin homeostasis and maintenance. Second, p63 is involved in the self renewal/ proliferation of adult epidermal cells, thus the disappearance of a fully stratified epidermis in such a short period of time between E15.5 and E18.5 - could best be explained by either a complete detachment of the tissue, or massive apoptosis. Accordingly, p63 directly regulates expression of extracellular matrix adhesion molecules, like the hemidesmosomal laminin5 , its integrin receptor $\alpha 6 \beta 4$ and the desmosomal chaperon protein PERP, and of a whole program of attachment. ${ }^{5}$ However, this issue remains to be determined.

In summary, although the overall outcome of the Brdm2 mice analyzed by Wolff et al. ${ }^{4}$ are not different from those originally described in the original Mills et al. ${ }^{2}$ paper, and later confirmed in several publications, the differences are numerous enough to potentially generate a considerable degree of confusion in the field. It is possible that the low p63 mRNA and protein levels were simply missed in the earlier papers, because of

${ }^{1}$ INSERM U898, Faculty of Medicine, Av. Valombrose, Nice 06107, France and ${ }^{2}$ Dipartimento di Scienze Biomolecolari e Biotecnologie, Università di Milano, Via Celoria 26, Milano 20133, Italy

*Corresponding author: R Mantovani, Dipartimento di Scienze Biomolecolari e Biotecnologie, Università di Milano, Via Celoria 26, Milano 20133, Italy.

Tel: + 3902 50315005; Fax: + 025031 5044; E-mail: mantor@ unimi.it 
technical reasons. However, because of the number of laboratories that made use of $\mathrm{Brdm} 2$, we consider this possibility unlikely. One study that evaluated p63 expression in oral epithelia and tooth placodes and hair follicles in wild type and Brdm2 KO is particularly illustrative: ${ }^{6}$ the authors analyzed mice from E11 to E16 and dramatic defects in dental placodes and hair follicle formation were evident. The dental lamina of thickened epithelia did form at E12-E13, but was blocked and later regressed. No additional epithelial differences with respect to the original report were detected in this study and p63 expression was negative by in situ hybridization. The phenotypic differences point to a more general question: are the partial $\mathrm{KO}$ mice analyzed by Wolff et al., ${ }^{4}$ identical to the Brdm2 mice originally described by Mills et al. ${ }^{2}$ ? There are reports of cellular clones in which the targeted allele has reverted back to wild type through spontaneous homologous recombination or, more likely in this case, removal of the duplicated locus. The event is rare $\left(10^{-6}\right.$ as reported in Zheng et al..$\left.^{7}\right)$ and patchy areas of normal tissue could coexist with the dramatic skinless phenotype; the $\alpha$-and $\beta$-isoforms were apparently not detected by Wolff et al., ${ }^{4}$ making the interpretation of a simple return to a wild-type configuration problematic.

There are other possible interpretations. Although inbred strains are stable, they are not fixed entities and phenomena of new mutations occur within mouse colonies. Genetic recombination at the p63 $3^{\prime}$-region, distant from the area analyzed in the paper, might have brought into proximity elements that increase the strength of the p63 promoters most likely the $\Delta \mathrm{N}$ - or confer greater stability to the mRNA(s) produced by Brdm2, stabilizing it and allowing the detection, and functional activity, of the p63 $\gamma$-like isoform. In humans, it has been known for decades that different deletions within clusters of duplicated genes occur and can have dramatically different outcomes: $\delta$ - $\beta$ thalassemias and deletional HPFH, for example, both have deletions in the $\beta$-globin gene, but in the latter, a genetic element with enhancer activity is brought close enough to the $\gamma$-globin promoter to maintain activity throughout the adult period. ${ }^{8}$ The transcriptional effect is not overwhelming, but sufficient to prevent $\alpha$-globin precipitation in the red blood cells, and hence thalassemia.
If a genetic event has modified the levels of $p 63 \gamma$, so that it becomes detectable, when - and possibly where - did this happen? This is really an important issue, as the Brdm2 mice were used in most experiments aimed at ascertaining the role of p63 in specific cellular functions and developmental processes. For example, whereas Brdm2 and McKeon's KO mice yielded apparently identical phenotypes as far as prostate development is concerned, ${ }^{9}$ other experiments performed in neuronal, germ cells and bladder development were reported only with Brdm2. Furthermore, there are differences in the outcome of tumors, when backcrossed with p53 KO animals. ${ }^{10,11}$ Might some of these experiments be influenced by the particular source of mice used? A recent genetic event would entail minor consequences in terms of the interpretation of past results. The Bradley laboratory made their mouse models available to the scientific community and they are also distributed by JAX: these colonies might be in an original Brdm2 configuration, allowing careful examination side by side - of the two phenotypes, through different phases of skin development, as well as the characterization of the molecular causes of the phenotypic changes.

Whether the original Brdm2 mice really express a functional p63 $\gamma$-like protein that produces a phenotype distinct from the other three p63-deficient mouse models, or whether the detailed observations of Wolff et al. ${ }^{4}$ represent spontaneous genetic events remains to be determined. Wolff et al. might have 'stumbled' on a new p63 model that could be important for analyzing the numerous functions of p63 in development and cancer.

1. Rinne T et al. J Med Genet 2006; 140: 1396-1406.

2. Mills AA et al. Nature 1999; 398: 708-713.

3. Yang A et al. Nature 1999; 398: 714-718.

4. Wolff $S$ et al. Cell Death Differ 2009; 16: 1108-1117.

5. Carroll DK, Brugge JS, Attardi LD. Cell Cycle 2007; 6: 255-261.

6. Laurikkala J et al. Development 2006; 133: 1553-1563.

7. Zheng B, Mills AA, Bradley A. Nucleic Acids Res 1999; 27: 2354-2360.

8. Forget BG. Ann NY Acad Sci 1998; 850: 38-44.

9. Grisanzio C, Signoretti S. J Cell Biochem 2008; 103: 1354-1368.

10. Guo X, Mills AA. Cell Cycle 2007; 6: 305-311.

11. Flores ER. Cell Cycle 2007; 6: 300-304. 